\title{
Endocytoscopic imaging and tattooing: a caveat
}

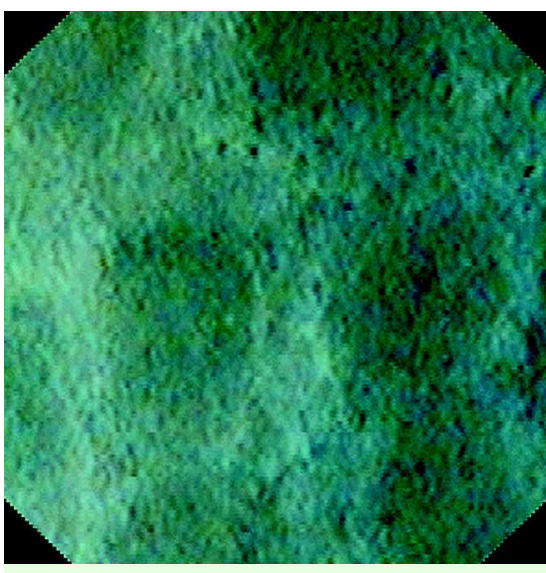

Fig. 1 Endocytoscopic picture $(\times 450)$ of colonic mucosa after tattooing. Crypts appear as yellowish structures.

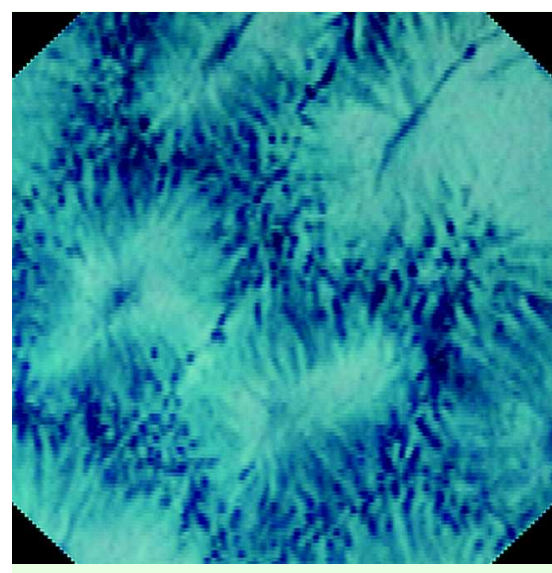

Fig. 2 Normal endocytoscopic image of colonic mucosa surrounding tattooed area.

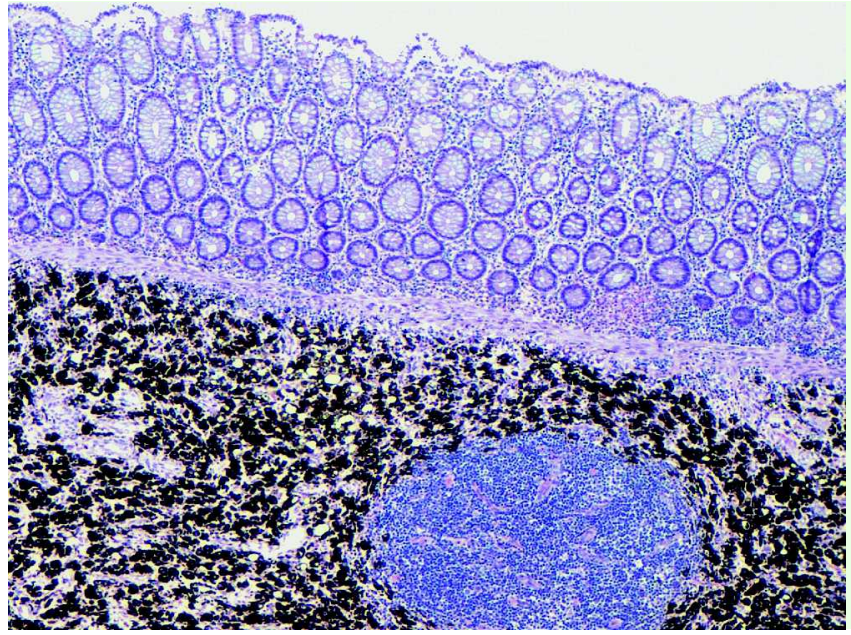

When precise anatomical location of a lesion is essential for endoscopic follow-up after resection, the colonic mucosa is commonly tattooed by submucosal injection of India ink [1 -3]. Our early experience [4] seems to suggest a role of endocytoscopy (ECS) for improving detection of early recurrences of high risk and/or malignant adenomas. However, potential imaging artifacts induced by previous tattooing led us to investigate limitations, if any, of ECS under these circumstances. Ex vivo ECS was performed in two patients who had undergone surgical resection of the colon for large adenomas, which had been marked preoperatively using India ink. A prototype Olympus XEC-300-U endocytoscope (Olympus Medical System Co., Tokyo, Japan) was
Fig. 3 Tattooed area that was initially examined using endocytoscopy (ECS), with hematoxylin and eosin (H\&E) staining $(\times 400)$. The mucosa is normal, whereas deposits of India ink pigment are evident in the submucosa. used according to the technique described previously [4].

In both cases we observed multiple brownish-yellow, blurred round structures, with regular borders, corresponding to colonic crypts within tattooed areas ( $\bullet$ Fig. 1). Compared with the normal endocytoscopic appearance of the surrounding mucosa ( $\odot$ Fig. 2), poor imaging of glandular structures made it nearly impossible to evaluate minimal changes and/or nuclear atypia, if any, of epithelial cells. The interstitial space was normally stained with methylene blue. Submucosal black staining was not visualized by ECS, as expected. The tattooed areas studied by ECS were then examined pathologically. Microscopic examination with hematoxylin and eosin (H\&E) re- vealed that the mucosa was normal whereas many black-stained macrophages were distributed throughout the entire submucosa and partially within the muscularis propria ( Fig. 3).

The ECS findings could be explained by the anatomy of crypts, whose deep branches through the mucosal layer get closer to the submucosal Indian ink. Black pigment is likely to interfere optically with the staining of crypts, whereas the interstitial connective tissue surrounding the glandular necks appears normally stained by methylene blue.

Poor imaging of glandular details may limit the use of ECS for surveillance examinations after tattooing. When ECS is scheduled, a modified tattooing technique [5] probably should be adopted.

\section{Endoscopy_UCTN_Code_CPL_1AJ_2AB}

\section{A. Fasoli ${ }^{1}$, V. Pugliese ${ }^{1,2}$, B. Gatteschi ${ }^{3}$,} M. Truini ${ }^{3}$, E. Meroni ${ }^{1}$

Department of Interdisciplinary

Endoscopy, National Institute for Cancer Research, Genova, Italy

Department of Oncology, Biology and Genetics, University of Genova, Genova, Italy

Department of Pathology, National Institute for Cancer Research, Genova, Italy

\section{References}

1 Park JW, Sohn DK, Hong CW et al. The usefulness of preoperative colonoscopic tattooing using a saline test injection method with prepackaged sterile India ink for localization in laparoscopic colorectal surgery. Surg Endosc 2008; 22: 501 - 505

2 Price N, Gottfried MR, Clary E et al. Safety and efficacy of India ink and indocyanine green as colonic tattooing agents. Gastrointest Endosc 2000; 51: $438-442$

3 Nizam R, Siddiqi N, Landas SK et al. Colonic tattooing with India ink: benefits, risks, and alternatives. Am J Gastroenterol 1996; 91: $1804-1808$

4 Meroni E, Gatteschi B, Fasoli A et al. Detection of tissue abnormalities in normal mucosa surrounding colorectal cancer using an endocytoscopy system. Endoscopy 2007; 39: 369- 370

5 Fu KI, Fujii T, Kato $S$ et al. A new endoscopic tattooing technique for identifying the location of colonic lesions during laparoscopic surgery: a comparison with the conventional technique. Endoscopy 2001; 33: 687691 
Bibliography

DOI $10.1055 / \mathrm{s}-0028-1119489$

Endoscopy 2009; 41: E41 - E42

(c) Georg Thieme Verlag KG Stuttgart · New York . ISSN 0013-726X

\section{Corresponding author}

\section{E. Meroni, MD}

Department of Interdisciplinary Endoscopy

Institute for Cancer Research

Genova

Italy

Fax: +39-10-5600645

emanuele.meroni @istge.it 